\title{
Developing sustainable Open Science solutions in the frame of EU funded research: the OpenUP case
}

\author{
Eleni Toli \\ Electra Sifacaki \\ Natalia Manola \\ Yannis Ioannidis \\ University of Athens \\ Athens, Greece \\ elto, esifacaki, natalia, \\ yannis@di.uoa.gr
}

Michela Vignoli

AIT Austrian Institute of Technology

Vienna, Austria

michela.vignoli@ait.ac.at

\author{
Tony Ross-Hellauer \\ Know-Center GmbH \\ Graz, Austria \\ tross@know-center.at
}

\author{
Edit Görögh \\ University of Göttingen \\ Göttingen, Germany \\ goeroegh@sub.uni-goettingen.de
}

\author{
Saskia Woutersen- Windhouwer \\ University of Amsterdam, The \\ Netherlands \\ s.windhouwer@uva.nl
}

\begin{abstract}
Open Access and Open Scholarship have revolutionized the way scholarly artefacts are evaluated and published, while the introduction of new technologies and media in scientific workflows has changed the "how" and to "whom" science is communicated, and how stakeholders interact with the scientific community and the broader public. The EU funded project OpenUP is connecting people, information and tools and provides a knowledge hub and a validated framework for the review, assessment and dissemination aspects of the research lifecycle, under the prism of a gender-sensitive Open Science.
\end{abstract}

Permission to make digital or hard copies of all or part of this work for personal or classroom use is granted without fee provided that copies are not made or distributed for profit or commercial advantage and that copies bear this notice and the full citation on the first page. Copyrights for components of this work owned by others than ACM must be honored. Abstracting with credit is permitted. To copy otherwise, or republish, to post on servers or to redistribute to lists, requires prior specific permission and/or a fee. Request permissions from Permissions@acm.org

OpenSym '18, August 22-24, 2018, Paris, France (C) 2018 Association for Computing Machinery. ACM ISBN 978-1-4503-5936-8/18/08 ...\$15.00 https://doi.org/10.1145/3233391.3233528

\section{CCS CONCEPTS}

- Information systems $\rightarrow$ Information systems applications; Collaborative and social computing systems and tools $\bullet$ Information systems $\rightarrow$ World Wide Web; Web searching and information discovery

\section{KEYWORDS}

Open science, research lifecycle, citizen science, open peer review, altmetrics, innovative dissemination, sustainability, pilots, policy framework, community platform

\section{ACM Reference format:}

E. Toli, E. Sifacaki, N. Manola, Y. Ioannidis, T. Ross-Hellauer, E. Görögh, M. Vignoli, V. Banelyte, P. Manghi and S. WoutersenWindhouwer. 2018. SIG Proceedings Paper in word Format. In Proceedings of The 14th International Symposium on Open Collaboration, Paris, France, August 2018 (OpenSym'18), 10 pages. https://doi.org/10.1145/3233391.3233528

\section{INTRODUCTION}

Open Access (OA) and other Open Science (OS) components, accompanied by technological developments, are changing the practices of stakeholders involved in academic research. Exponential growth of research outputs and an increasing demand for more open, transparent and reproducible science has been pushing stakeholders 
(including researchers, publishers, funders, institutions and industry) to rethink how research results are reviewed, published and assessed.

Innovative tools to disseminate research have altered how and to whom science is communicated, and how the public Innovative tools to disseminate research have altered how and to whom science is communicated, and how the public interacts with the scientific community. In addition, traditional research review and evaluation methods do not correspond to this changing landscape and require new policies and practices.

Through analysis, consultation, hands-on engagement with researchers, publishers, institutions and funders, industry and citizens, the EC-funded OpenUP project (http://openuph2020.eu/) is working to a) define a framework that defines roles and processes, benefits and opportunities, b) validate the proposed mechanisms through a series of pilots involving researchers from four scientific communities (Life Sciences, Social Sciences, Arts \& Humanities, Energy), and c) come up with practical policy recommendations and guidelines, addressing also gender-related issues in science, to be used by EU, national and institutional policymakers at different settings. OpenUP is engaging with all stakeholders via a series of outreach and training events, and the creation of the OpenUP Hub (https://www.openuphub.eu/), a collaborative web based Knowledge Base that hosts a catalogue of open tools/services, methodologies, best practices from various disciplines or settings, success stories, and reports. This increased level of engagement and knowledge will feed into the development of research and innovation policies that aim to support and complement a gender-sensitive Open Science.

With a clear trend towards open scholarship, OpenUP has engaged partners with a proven outreach capacity in all aspects of the review-disseminate-assess cycle: research organizations and universities that have a track record on open science policies, academic libraries, scholarly publishers, e-Infrastructures, public policy consultancy SME.

\section{BACKGROUND}

The cyclicity is an important element of science and research. In the recent years the term research lifecycle has been established, referring to the cyclical research processfrom idea to implementation to dissemination and back to idea [5], including all intermediate steps: planning, primary research, development, publishing, review, assessment, preservation, dissemination, reuse.

In this process, three steps stand out, as they provide the conditions for bringing science from the "laboratories" to the society: the review, the assessment and the dissemination of research results. All three require a high level of interaction either with other peers or broader communities, include multiple processes, and are necessary for making research results available, especially in the context of Open Science. It is for these reasons that the OpenUP project focuses on these three pillars working on new tools and policy recommendations across them.

For many years, the perception of how scholarly artefacts should be evaluated, published and assessed has been rather conventional, with several problematic aspects: The traditional peer review process used by many scientific journals is subject to criticism on several fronts. It has been described as unreliable, too lengthy, biased and lacking accountability and transparency [20, 21]. Research dissemination, often implied a one-way communication from researchers to the society, or was directed only towards other academics. The society, for whose benefit research is done, had merely to "consume" the research results, with no real possibility to provide feedback and have a stake in this process. Evaluation methods of research and researchers are facing criticism as well. The currently used bibliometric indicators like the h-index [17] or the Journal Impact Factor (JIF) [12] are believed to have a bias towards senior researchers or reflect the overall impact of a journal and not that of a specific article [16, 29]. These and other bibliometric indicators are failing to prove their suitability for measuring research outputs and their impact, in the context of a movement towards Open Science. They also do not consider other types of activities that scientists engage in, for example review work.

Open Science and new digital-networked technologies are now revolutionizing all processes above. They have also changed the requirements and practices of a range of different stakeholders, including researchers, publishers, funders, institutions, industry and the public. The traditional foci of publication and evaluation do not satisfy the needs of this changing landscape, and the understanding that it is time for these practices and processes to open up gains momentum.

Open peer review (OPR), with its different components, opens up the traditional process in order to help address the concerns mentioned before. OPR introduces such aspects as open identities (author and reviewer disclose their identities), open reports (review reports are published along the publication) and open participation (wider participation of interested parties in the process) [9]. These new aspects enable a more open discussion around research outputs. OPR can also help authors get feedback not only on the final research outputs (articles or books) but also on what is created at different stages of the research process (software codes or datasets).

Social media and other web-based technologies provide wider platforms for researchers to innovatively disseminate their work and for public and other parties to get information or engage with the researchers. Many research funders are also calling for stronger engagement of policy makers, industry, civil society organisations and citizens [11]. Such 
engagement of different stakeholders in research and innovation is needed to foster mutual understanding, cocreate research and innovation outcomes, provide input into policy agendas and ultimately to facilitate research result uptake.

In response to this, altmetrics emerged to broaden research and researchers' evaluation and impact. The growing uptake of new forms of dissemination (e.g. blogs, Twitter, openly available reports and data) is now also driving the use of alternative metrics. They hold the potential to improve the way research impact is understood and measured as well as to identify gaps in the researcher recognition, evaluation and researcher career advancement systems that have been previously overlooked. The concept of altmetrics is, however, still new and rather a small proportion of researchers are aware of it and even fewer use them [27].

\section{OPPORTUNITIES AND CHALLENGES IN UPTAKE}

Open Science is undoubtedly in uplift, and this is reflected in the significant increase of related outcomes: OpenDOAR, for example, listed in May 20163.090 open access repositories [23, 24]. Flagship initiatives initiated or endorsed by the European Commission have also greatly contributed to it. The seventh framework strongly encouraged for the first time the publishing of research data and publications in open access repositories, through the Open Data Pilot initiative. In H2020 this direction has become even more "mainstream" as research projects now had to justify the opt out from open data publishing. A new dimension has been added to the discussion: "how to get the most out of the open data published, by making them findable, accessible, interoperable and reusable (FAIR)" [31]. In parallel, expert groups and working groups have been established by the European Commission to produce recommendations for several areas of Open Science [8]. This is an environment that triggers also further developments in OPR, innovative dissemination and altmetrics. The OpenUP landscape scanning showed that although the new methods and tools have been taken up mostly by researchers and other actors in the academic world, several reservations still exist. Some of the arguments researchers brought up, when asked about the adoption of novel practices in relation to review, assess and dissemination include the following: they still feel tied up to the prevailing system of the peer review, dissemination and evaluation practices. The new concepts and practices are not well-established, and some researchers and other stakeholders are not aware of the new developments and what specific options they entail. Adoption of novel methods also requires time and financial resources. This might compromise the time and other resources researchers want to devote to conduct their scientific investigations.
These considerations explain why alternative practices are not as widely adopted as they could and have not yet scaled out, despite the impact and benefits they could have, and which is recognized by the majority of stakeholders, as this has been also captured in a broad survey conducted by the OpenUP project. To overcome this barrier, the EC and national governments are monitoring related developments also through the establishment of experts groups and the funding of various projects that aim to study and test the novel approaches. It is exactly in this context OpenUP project is unfolding its activities, aiming to contribute to the paradigm shift towards open science.

\section{PROJECT METHODOLOGY}

Ascribing to Open Science principles reflects first and foremost the commitment to make scientific knowledge more transparent and accessible, also by opening up the process to broader communities and society. The emerging of Citizen Science, we witness in the recent years, makes this process a bi-directional one: citizens are no longer only the recipients of research results, but play an active role in how research is done. Their involvement offers whole new possibilities to scale out research results, offers a means of doing substantial, thoughtful public outreach and tackle "otherwise intractable, laborious or costly research problems" [15]. Citizen Science is a way of democratizing science [19].

To our understanding, the above set a general framework for the work plan of an Open Science research project: it should pursuit community driven objectives and adopt a participatory design. The OpenUP project is following this direction to ensure that the needs, concerns and ideas of stakeholders involved in the review-assess-disseminate steps of the research lifecycle are heard and respected. The OpenUP partners are engaged in an overarching research exercise, implemented on two parallel and complementary levels:

1. Activities and tools for the direct interaction with communities and individuals. These include interviews, focus groups, training events, policy workshops, conferences and most importantly the operation of seven pilots. All the activities above play a significant role in the project, as they provide the necessary mechanisms to establish a "real" network of people and allow the direct collaboration and exchange.

2. Tools and services for the online interaction. This refers to the online collaboration platform OpenUP $H u b$ and the conduction of an online, Europe-wide survey of researchers on the key topics of the project. The Hub allows for a constant interaction and information flow, while through the survey we reached out to the main experts and stakeholders to 
gather their inputs on the current practices, challenges and the latest developments.

In addition to the above, our work is underpinned by broad landscape scans of literature and national policies on open peer review, innovative dissemination and altmetrics. All project's results are being tested in the OpenUP pilots involving researchers from four scientific communities. All the findings and conclusion of OpenUP will be analysed through a scenario analysis. The main results and selected scenario will also be validated in a high-level policy workshop involving representatives from national and EU level policy-making bodies, institutions, funders, researchers and infrastructure providers. The final product of the project will be evidence-based and validated recommendations directed to the EU, national and institutional policy-makers, funders and researchers looking for ways to address the emerging challenges and adapt to the rapidly changing scientific research landscape.

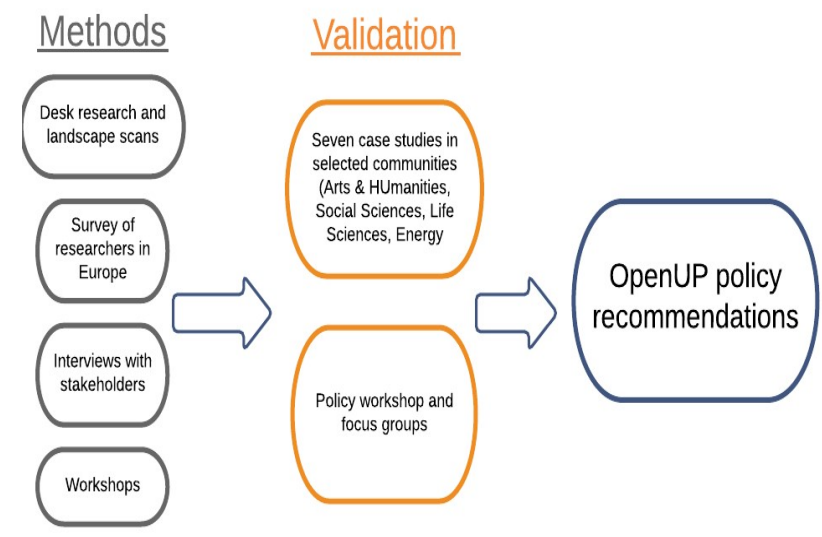

Figure 1: The methodological framework of OpenUP leading toward the stakeholder-validated policy recommendations.

In the remaining sections we present major findings on the review-assess-disseminate aspects, and results achieved thus far, as several project activities, and especially the pilots and the OpenUP Hub, are still ongoing. We also make an outlook on / to the expected policy recommendations and the sustainability of the project outcomes. researchers are aware of it and even fewer use them [27].

\section{THE OPENUP PILOTS}

\subsection{Pilots methodology and importance}

The aim of the OpenUP pilot studies is to test and evaluate selected innovative peer review, dissemination, and impact measuring approaches applied to specific research areas and communities. Together with research communities from arts and humanities, social sciences, energy, and life sciences, OpenUP applies and tests technical and processual solutions for innovative peer review, dissemination, and impact assessment. The pilots are two design studies (pilots 2 and 3); four workflow implementations (pilots 1, 4, 5 and 6), and a statistical analysis (pilot 7). Insights gained from the evaluation of the individual pilots will deliver further input on working practices, developing standards, and remaining gaps. In terms of awareness raising and community support, the pilot studies strive to document resulting success stories and working practices, which can become a useful resource for other communities. Finally, OpenUP hopes to inspire and equip the communities directly involved in the pilot studies with knowledge and methods to adopt the tested Open Science practices beyond the duration of the project. The final evaluation report will summarise implications and lessons learned from the three key areas of OpenUP and provide useful input to the policy recommendations. Major findings from the interim report [30] are summarised here. value and the sample history.

\subsection{Major interim findings}

Pilot 1, Open Peer Review for Conferences: Initial feedback from the researchers involved in the OPR process at the EMVA [10] conference was very positive. Overall, the participants expressed a strong acceptance of the proposed OPR process [32]. The greatest fears associated with OPR included: biased/whitewashed reviews due to nonanonymity; and backlash for bad reviewing (e.g. over other channels/private email).

Pilot 2, Open Peer Review for Research Data: HMD [14] is a well-known data source and is being analysed by OpenUP to provide insights on best practices that can be adopted also by other social science communities. Interviews with key experts at HMD contributed to a better understanding of the data management procedure in population studies in general and the usage of the HMD in particular.

Pilot 3, A data journal for the Arts and Humanities: The pilot develops a framework for a Humanities data journal which will be implemented by DARIAH [6]. Stakeholder feedback and best practices of how data publishing is being approached and managed in Arts and Humanities was gathered from earlier surveys and reports as well as in interviews with experts.

Pilot 4, Transferring the research lifecycle to the web: The Open Online Research (OOR) tool [4] has been tested by various groups of participants. The results confirmed that the tool enables online collaborative interpretation. A core observation is that the whole process and tool need to "radiate" diversity and inclusiveness. Differences in terms of gender, ethnicity, educational background and hierarchy are influencing participation in general and the kind of contribution in particular.

Pilot 5, Reaching businesses and the public with research output: We conducted interviews with science communication experts to elicit dissemination requirements 
by the two targeted audiences (businesses and general public). Information on needs and expectations of businesses and the general public audiences, as well as the science communicators' experiences and success stories were collected and fed into communication guidelines for researchers [7].

Pilot 6, Reflexivity of metrics on medical research and dissemination practices: The translational research community we are cooperating with is highly interested and open to open science and Altmetrics, in particular the institutional managers. The community needs to develop novel criteria and categories of what makes biomedical research more transparent and efficient, for which we aim to provide some support.

Pilot 7, Piratical demand as a form of impact indicator and reaching unexpected audiences: We scraped library availability and metadata from the worldcat database for more than a million titles. For each title, we scraped for more than a dozen locations (as defined by the most frequent locations of illegal downloads), prices and legal availability. We developed an online service allowing real-time exploration of the dataset.

\section{THE OPENUP HUB}

It is an auspicious fact that there is a plethora of initiatives advocating and developing tools for the Open Science movement, contributing every day to the wider adoption of its principles. They range from very broad ones, to others that address only particular aspects. Some are providing an e-learning platform with training resources on open science aspects and guidance on how to develop related strategies and skills (https://www.fosteropenscience.eu/). Some others advocate for performing responsible research and innovation (RRI), provide a toolkit to support it and aim at building a European community of practice around this topic (rritools.eu). Others support the reproducibility or research artefacts and data by providing services for integrating Persistent Identifier (PID) services across the research lifecycle and data publishing workflows (https://projectthor.readme.io). And there is of course the most significant initiative OpenAIRE (https://www.openaire.eu/), the broadest European network of people, dedicated to the promotion of open scholarship and the substantial improvement of the discoverability and reusability of research publications and data. It implements and aligns Open Science policies across Europe and the world, deploys services to embed Open Science into researcher workflows, develops global open standards for linking all research, and monitors Open Science in Europe.

These are only few examples of the numerous projects and initiatives that are active in the area in the recent years, showcasing the different possible directions. The OpenUP Hub is contributing to this open science landscape through two key features: while it reaches out to all stakeholders involved in the research lifecycle (researchers, universities, research institutes, European and international organisations, librarians, funders and ministries, content publishers) it maintains a strong focus on only the three elements "review-assess-disseminate". It aims thereby to create an increased level of engagement, grounded on the bidirectional interaction with the community, providing a comprehensive knowledge base around these topics that can support e.g. early stage researchers in their work, while offering in parallel the tools for a direct collaboration.

\subsection{The community dimension: ensuring multi-way communication}

OpenUP Hub (openuphub.eu) is an open, dynamic and collaborative knowledge environment that systematically captures, organizes and categorizes research outcomes, best practices, tools, and guidelines relevant to reviewdissemination-assessment phases of the research lifecycle through the prism of Open Science. It is a community-driven solution which puts the scientific community in the center to support its needs. It primarily addresses all key stakeholders engaged in the research lifecycle: researchers, young scholars, educators, librarians, publishers and Research \& Innovation $(\mathrm{R} \& \mathrm{I})$ project members. It also actively engages the rest of its important stakeholders that have a "stake" in the research lifecycle: Open Science advocators, policy makers, funders, general public and representatives from the industry.

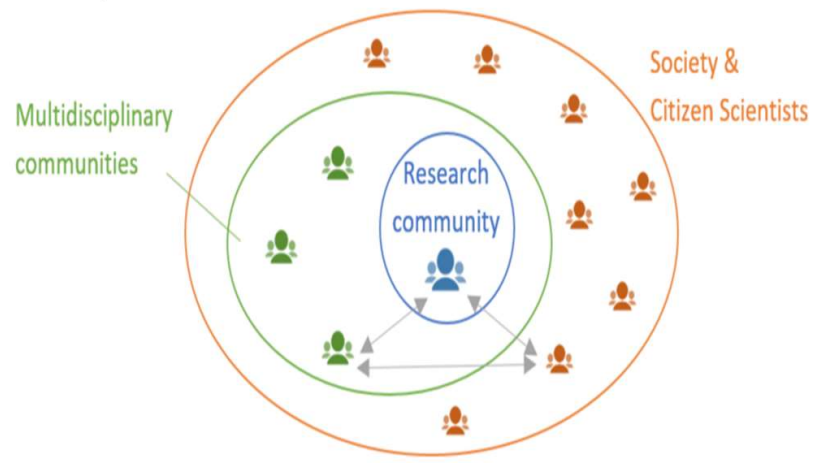

Figure 2: Interaction triangle within different research groups, engaging the society and communicating to and with the society [18].

The multi-way communication and interaction are an integral part of the OpenUP Hub. It is a tailor-made solution designed by the community for the community, that allows people interested in or working on innovative methods of review-assess-disseminate to connect to each other and publicize their work. The interaction in a higher level schema can be therefore described through a triangle of a homogenous research community, multidisciplinary scientific communities and the society/citizen scientists, as 
illustrated in Figure 2, where all edges denote two-way communication.

\subsection{Functionalities and use}

OpenUP shifts from one-way to multi-way communication and open dialogue, opening multiple channels that give the opportunity to all users to interact with the hosted material and connect with other users. Interaction is encouraged and supported by several means. The OpenUP Hub provides:

- A toolbox for open science solutions, trainings and services addressing specific needs \& questions on alternative peer review, altmetrics and innovative dissemination methods. The hosted material is selected based on a thorough landscape scan of the three domain fields and validated by the community.

- A Blog where articles and point of views on Open Science are collected, uploaded and promoted. Scientists, librarians, open science advocates and all community members are motivated to promote and advance their work, share innovative ideas, receive feedback from experts and interact with other community members.

- The Q\&A forum Ask Open Science [2] to promote the immediate communication and chatting between members, ask questions or provide answers and support to other members on issues related to Open Science. The Q\&A forum The Ask Open Science has been established in liaison with Christian Pietsch and supported by the Bielefeld University

- An Observatory that senses the community pulse about open peer review, altmetrics, communication of science, the role of women in science and responsible research \& innovation.

- A Calendar to share and view major events of the field.

- A Suggestion Box, where all users can suggest new tools, material, events, ideas.

These features are the result of the analysis of our interaction and exchange with the communities. Being a communitydriven initiative, the OpenUP Hub is open to adapt and change, so that it remains a useful tool for the review-assessdisseminate aspects and allows people working on, or interested in these topics to connect to each other, publicise their work and create a community of peers.

\section{MAJOR FINDINGS ON THE THREE PROJECT PILLARS}

\subsection{Review}

The alternative review tools, services and approaches offer various methods for review (e.g. open review, prepublication or post-publication review, collaborative or decoupled review, and different degrees of openness in identity, participation and interaction among stakeholders). They might differ in their solutions, but they all carry several common features: (1) they move away from the established publishing and review system by finding solutions to the problematic aspects of the traditional single/double blind review process (i.e. lack of transparency, potential bias, quality of review, etc.), (2) the review process becomes more transparent either by opening up certain aspects of the process, or by providing detailed review policies, (3) they urge a more conscious, collaborative participation by stakeholders, either through invitation and dialogue within small circles between authors, editors and reviewers, or through crowdsourcing the process and allowing the public to add comments and reviews.

These tools and services described on the basis of the seven attributes of open peer review [26] identify the main issues where intervention is needed in the traditional review system. The solutions they offer invigorate conversation among researchers about the functionalities of review as well as their role and responsibilities in the process. Such dialogue, which is continuously reshaped by the exchange of ideas, new perspectives (open science approach) and tools (e.g. ORCID review tracking functionality) and emerging frameworks (e.g. pre-registration of research, uploading preprints for grant application), promises a more scholarcentric approach. The peer review discourse is also advanced by a more proactive stance of the stakeholders involved. Initiatives, such as the Open Science Peer Review Oath by F1000 Research [1] or the Peer Reviewers' Openness Initiative [25], urge reviewers to define the terms of review and ensure that reviewed scientific results are open and reproducible, which consequently lends transparency to the peer review process and increases its impact and outreach. The OpenUp user-centered survey results - conducted in the beginning of 2017 to test the researchers' attitudes towards peer review tools and services [27] - show that researchers seem to be reluctant to fully embrace openness in the review process, but definitely see advantages of a transparent, collaborative review process. In order to make researchers less vulnerable to share their work and make their research open for comments, these alternative tools and services would benefit from further standardization and integration into the research cycle [28]. However, the formal acknowledgement of the viability and validity of these alternatives, such as independent review services or review solutions for repositories and preprint servers, presupposes discussion on their sustainability, long-term availability, and their uptake by the researchers.

Fiona Godlee [13] cited four main reasons in support of open peer review: (1) ethical superiority - open peer review makes the reviewer and the editor more accountable for the peer review process, (2) lack of adverse effects, (3) feasibility, and (4) recognition for peer review work. More 
than a decade later these benefits still seem as valid basis for the increasing presence of review alternatives. Our survey results support this view since researchers consider transparency and the recognition, alternative review services and methods offer, valuable aspects of the changing scholarly review discourse.

\subsection{Disseminate}

Our exploration of the emerging landscape of innovative dissemination has been driven by user-needs. The goal is to categorise stakeholders in order to provide researchers with entry points to innovative dissemination where methods and tools can be chosen based on audiences, their skills and their requirements. Our stakeholder groups include researchers, institutions, funders, publishers, and citizens.

To assess user-needs and scope existing tools and services, we have employed three primary instruments: (1) a literature review, (2) an extensive survey of European researchers, (3) targeted stakeholder interviews, (4) case studies of projects employing innovative dissemination methods, systematic review of online tools and platforms.

Our investigation has revealed a wealth of approaches going beyond traditional academic publishing and gathered success stories and good practices from all over the world. One of the most striking lessons learned from the analysis is the fact that dissemination in an open science context is increasingly done at earlier stages of the research lifecycle and is thus becoming an integral part of the whole research workflow. In addition, dissemination becomes more interactive; as a result, it is often difficult to draw the line between the activities of dissemination and participation.

With respect to gender, we have identified four relevant aspects: (1) the gender distribution within the team responsible for research and dissemination, (2) representation of gender in the disseminated materials, (3) gender sensitivity and inclusiveness of the used dissemination tools, platforms and strategies, and (4) gender aspect related to the target audience. When it comes to openness, the results of the case studies show that researchers need guidance in terms of choosing the right license for their materials in order to achieve the highest possible reuse of their materials.

When targeting the general public, care must be taken to break down this very homogenous group into smaller groups of potentially interested parties. It is important to transport clear and exciting information snippets and to tell a story. While we can observe enthusiastic uptake with specific groups of researchers, the survey results also showed that there is a gap in practice when it comes to innovative dissemination. Communicating to a wider audience seems to be more of a developing norm with early adopters than a widely exercised practice. Lack of knowledge of innovative dissemination channels and methods are important barriers for adoption, especially for young researchers. This is where
OpenUP's innovative framework comes in. It is designed to give stakeholders recommendations for innovative dissemination practices tailored to their requirements and their resources. To this end, the OpenUP Hub has developed explanatory resources to brief stakeholders on key concepts, as well as directories of key tools and services to enable innovative dissemination.

\subsection{Assess}

The main goal of our research work in regard to alternative assessment techniques, is proposing a validated taxonomy of channels of scientific knowledge dissemination and altmetrics. The taxonomy provides scholars and academia in general, the possibility to choose the relevant indicators based on the communication channel selected to disseminate the underlying research.

This endeavor involves a broad bottom-up sourcing of information and intelligence about how both dimensions will be linked. In particular, we have put to work: (1) a literature overview on altmetrics and indicators to assess impact of research (2) interviews with experts from the field of bibliometrics and altmetrics and (3) a desktop survey to investigate the adoption rate of altmetrics indicators. Our key findings [3] are:

- The survey results indicate that traditional channels of dissemination such as publications and conference presentations, are still in a high preference of researchers for communicating their results. As a result, the adoption rate of alternative metrics remains in a primitive stage, introducing the necessity for training in deploying innovative dissemination strategies, using alternative techniques and assessing these channels.

- Different research fields have different needs and objectives in concern to dissemination which subsequently also influence the use of these channels. Therefore, the need for field specific interpretations and targeted solutions for assessment become necessary.

- According to the taxonomy results, it is observed that scholarly appreciation and altmetrics' coverage are highly correlated.

- Technical characteristics and lack of accessibility (e.g., existence of APIs) of innovative dissemination constrain usage and impede construction of metrics.

- More efforts are required to make metrics more "meaningful", namely, it is necessary developing a solid context and presentation of the data that produce them. This will be a provide more information for all parties of interest (publishers, scholars, policy makers, and service providers). mutual endeavors 


\section{LOOKING AHEAD}

In the remaining months of the project it is planned that the project will deliver its key outcomes that have the potential to impact the work of Open Science communities: we will analyse the outcomes of the seven pilots and combine them with the input received through the focus groups, we will provide peer review recommendations for the research workflow, we will expand content and functionalities of the OpenUP Hub and, finally, focus on the convergence of all activities and the production of a comprehensive policy framework. value and the sample history.

\subsection{Open review of scientific process}

Peer reviewing the whole research flow is certainly the most complete conception of evaluation and assessment of digital science. Its modeling includes the scientific method, which corresponds to how science is performed (the structuring of scientific thinking); and the experiments, which model how science was carried out in terms of "steps", performed using e-infrastructure tools (e.g. services, facilities), and input and output research products (e.g. datasets, algorithms, software). Existing approaches nicely address some of these challenges, but none of them tackles the general problem. The most advanced solutions are focused on encoding experiments as digital objects (e.g research objects), thereby targeting reproducibility of one specific step (typically the conclusive one) of the research workflow, hence they disregard the overarching issue of research flow peer review. After investigating these issues, the project has developed some ideas in the direction of the definition of a framework for the representation of "research flow peer review by scientific discipline". The framework introduces primitives to design templates of research flows, which can be followed by scientists to perform and peer review the scientific method. Ideally, such a framework may become the driver for developing tools for "ongoing peer review" of research flows, by "real-time hooking" to the underlying digital laboratory, where scientists are carrying out their research flow. Such tools would abstract over the complexity of the research activity and offer user-friendly dashboards to examine the scientific process adopted, explore the ongoing research flow, and evaluate its intermediate experiments and products.

\subsection{Outlook to the OpenUP Policy Framework}

The OpenUP policy framework is based on a solid research and evidence base that was built up by various research activities as well as by bringing stakeholders into an open dialogue to consensually identify and validated the reviewdisseminate-assess mechanisms fit for the evolving practices of RRI in an Open Science and gender sensitive context. The main groups of interest that will be addressed with this activity are institutional decision-makers, national and EUlevel policy makers, funders, libraries and infrastructure providers. The interim recommendations of our work are summarized as follows:

- Delivering awareness-raising activities, to researchers to become familiar with the challenges and the notion of review-dissemination-assessment phases of the research lifecycle underlying gendersensitive issues.

- Adapting policies and funding requirements to accommodate the needs of researchers when they engage in open peer review and innovative research dissemination. Providing sufficient time and financial resources for such activities through dedicated support actions, acknowledging them in assessments.

- Stimulating practice of new methods, facilitating collection of data and evidence, and playing an active role in the construction of a knowledge base regarding these practices.

- Sharing the results and findings of the successful initiatives at high-level and disseminating them to other interested parties, Europe-wide. Infrastructures should be developed to enable use also by countries with lower research budgets. European Union and national policy-makers should implement the best approaches and introduce them into research policies also considering gender aspects.

- Informing researchers and research administrations about green and gold Open Access, publishing procedures and possibilities to cover related cost, by organising inhouse trainings, as it seems that there is still a need for clarifications in this respect.

\subsection{Sustainability of projects results}

The project-based research has become the dominant way of doing research in Europe in the recent years. The huge number of projects funded through the several EU frameworks (currently 533.805 on CORDIS) demonstrate this. This form of doing research is a highly debated issue, with many supporters and opponents. It holds a truth, it is difficult to tackle, especially complex, scientific challenges, working on a short-to medium term timeframe. The danger exists, that the research objectives and goals are adapted, to be manageable and reachable within the limited time. On the other side of the coin, we have to consider that for many disciplines and countries, the EU funding is the most important resource they can rely on (if not the only one...) for performing research. This fact poses additional challenges to and increases the importance of the sustainability of the project outcomes.

Sustainability is an important concept for society, economics, the environment, and for science. As sustainability science has become a research field on its own, it shows that this issue is an integral part of research 
developments. The goal of sustainable science is to create long-term integrity of knowledge creation, discovery and dissemination (citation?).

Having in mind the above, OpenUP has been working on the sustainability aspects following the pattern: aggregate in the long-term people, data and online services under the umbrella of a validated policy framework. All them combined, reach a different quality and are more than the sum of the parts.

OpenUP has reached out, via the multi-faceted activities described in previous sections, to numerous Open Science stakeholders, most of them being researchers. This network of people has been created on the basis of shared research interests: OpenUP has engaged with them having the genuine interest to capture their views and needs on reviewassessment-dissemination, and offers in return customized information and services, and drafts related policy recommendations. Maintaining this network is certainly a challenge, which can only be achieved, if the tradeoff continues. This means for example, that the OpenUP Policy Framework should be considered only as the first draft provided by the project. Its extension and continuation lie in the hands of the community. Should they find a value in it, they will probably want to have a stake in its evolvement. In this respect, the OpenUP Hub has to play a major role.

The Confederation of Open Access Repositories (COAR) published five prerequisites for sustainable knowledge commons among them are the strengthening of local institution-based services, the connection of local services to regional, national and global networks, and the adoption of principles that ensure that the knowledge commons reflect the needs of the global research community. Thus, for the sustainability of a knowledge infrastructure, it is vital to work towards its integration into the global knowledge network and to include the community aspect right from the beginning.

Following the above line of thoughts, the strong community orientation has been an integral part in the development of the OpenUP Hub:

1. It is an end-to-end tool, from researchers to researchers, that includes services that add value to their work, supporting dissemination and feedback, are customized based on the main stakeholder profiles taking part in the review-assessmentdissemination phases, allows interaction between different communities and individuals.

2. It builds awareness and recognition on Open Science in general and towards the resource itself, which are important aspects that determine the sustainability of an Open Educational Resource (OER).

3. It makes the connection between "the virtual and the real worlds" using the Hub services also in physical meetings and trainings, in an effort to impact scholarly communication on a local level and connect virtual and physical communities.

4. It builds synergies and establishes permanent collaboration with other ongoing initiatives and projects in the area (e.g. OpenAIRE, FOSTER, RDA, Open Knowledge Maps, University of Bielefeld, Open Knowledge). This includes content and services exchange and mutual promotion.

In parallel to the above, the next implementation steps are being planned, including short and longer-term objectives. An immediate target is to increase the community generated content in comparison to the project generated or aggregated part, as this will be an indicator of the acceptance of the effort. The consortium is also discussing the next steps, that mainly focus on the operation of the hub after the official closing of OpenUP and how the community related elements above can be exploited.

Having the understanding that our knowledge hub is part of a commons ecosystem, we are considering the adoption of Open Business Models to ensure its sustainability. These can be additionally combined with a system of incentives for participation, for example by introducing a reward system based on gamification elements, and/or for contribution, for example through the creation of a Community Editorial Board. Working on the maturity of these ideas will be one of the major tasks for the OpenUP partners in the next period.

\section{CONCLUSION}

OpenUP partners have mapped the current reviewdisseminate-assess practice landscapes, measured researchers' attitudes and practices in these areas and collected expert views on recent and future developments. In addition, the results were cross-checked and validated in several academic communities through pilots as well as through workshops engaging librarians, funders, service providers, policy makers and other stakeholders. It has set up a knowledge and collaboration hub, which aspires to become a reference point in review-assess-disseminate topics.

OpenUP doesn't seek to cover Open Science in a horizontal way, but rather concentrates on important phases of the research lifecycle (review-assess-disseminate), providing tools, solutions and information about them, while connecting people working on, or interested in these topics. Driven by a community oriented methodology, OpenUP will codesign and validate with Open Science stakeholders a policy framework that is fit to support an open and gendersensitive ecosystem.

\section{ACKNOWLEDGMENTS}

OpenUP is a project that has received funding from the European Union's Horizon 2020 research and innovation programme under grant agreement No 710722 . 


\section{REFERENCES}

[1] Aleksic Jelena, Alexa Adrian, Attwood K. Teresa, Hong C. Neil, Dahlö Martin, Davey Robert, Dinkel Holger et al. 2014. The open science peer review oath. F1000Research 3. doi: 10.12688/f1000research.5686.1

[2] Ask Open Science. 2018. Retrieved March 15, 2018 from https://openscience.uni-bielefeld.de/

[3] Blümel Clemens. 2017. Report on final taxonomy linking channels of dissemination and altmetrics indicators, OpenUP D5.4 Deliverable

[4] Bröer Christian, Moerman Gerben, Casper W. Johan, Malamud Liza R., Schmidt Lianne, Stoopendaal Annemiek, Kruiderink Nynke, Hansen Christina , and Sjølie Hege. 2016 Open online research: Developing software and method for collaborative interpretation. In Forum: Qualitative Social Research, vol. 17, no. 3. Freie Universität Berlin.

[5] Castro G. Gabriela, Eldermire Erin , Tang Neely, Tancheva Kornelia in Association of College and Research Libraries. 2017. The Research Lifecycle and the Future of Research Libraries: A Library of Apps. At the Helm: Leading Transformation: The Proceedings of the ACRL 2017 Conference, March 22-25, 2017, Baltimore, Maryland, edited by Dawn M. Mueller. Chicago: Association of College and Research Libraries.

[6] DARIAH. 2018. Website. Retrieved March 7, 2018 from https://www.dariah.eu/

[7] Draft guidelines for communicating research to businesses and the general public. OpenUP. 2018. Retrieved March 7, 2018 from https://docs.google.com/document/d/1 mZM9kNgjBTL5kti9jgMi 8U2nDF2WYeKrmGv-33PeMZ0/edit?usp=sharing

[8] European Commission. 2018. Open Science. Retrieved March 10, 2018 from https://ec.europa.eu/research/openscience/index.cfm\#

[9] European Commission. 2015. Validation of the Results of the Public Consultation on Science 2.0: Science in Transition. Retrieved March 5, 2018 from https://www.eesc.europa.eu/resources/docs/validation-of-theresults-of-the-public-consultation-on-science-20.pdf

[10] European Commission. 2012. Responsible Research and Innovation: Research and Innovation Europe's Ability to Respond to Societal Challenges. Retrieved March 5, 2018 from https://ec.europa.eu/research/swafs/pdf/pub_public_engagement/r esponsible-research-and-innovation-leaflet_en.pdf

[11] European Machine Vision Association. 2017. Retrieved March 7, 2018 from http://emva-forum.org/second-forum.html

[12] Garfield Eugene. 1972. Citation analysis as a tool in journal evaluation. Science 178, no. 4060: 471-479. doi:10.1126/science.178.4060.471

[13] Gura Trisha. 2013. Citizen science: amateur experts. Nature 496, no. 7444: 259-261. doi:10.1038/nj7444-259a

[14] Godlee Fiona. 2002. Making reviewers visible: openness, accountability, and credit. JAMA, 287(21):2762-2765. doi:10.1001/jama.287.21.2762

[15] HDM. 2017. Website. Retrieved March 7, 2018 from http://www.mortality.org/

[16] Hicks Diana, Wouters Paul, Waltman Ludo, De Rijcke Sarah, and Rafols Ismael. 2015. The Leiden Manifesto for research metrics. Nature 520, no. 7548: 429. doi:10.1038/520429a.

[17] Hirsch E. Jorge. 2010. An index to quantify an individual's scientific research output that takes into account the effect of multiple coauthorship. Scientometrics 85, no. 3: 741-754. doi:10.1007/s11192-010-0193-9.

[18] Icon for "to share" from the Duotone series by Carol Liao. 2018. Retrieved January 15, 2018 from http://www.toicon.com/. Licensed CC-BY.

[19] Kullenberg Christopher, and Kasperowski Dick 2016. What is citizen science?-A scientometric meta-analysis. PloS one 11, no. 1: $\mathrm{e} 0147152$.
[20] Lee J. Carol, Sugimoto R. Cassidy, Zhang G. and Cronin, B. 2013. Bias in peer review. Journal of the Association for Information Science and Technology 64, no. 1: 2-17. doi:10.1002/asi.22784

[21] Manchikanti Laxmaiah, Kaye D. Alan, Boswell Mark and Hirsch A. Joshua. 2015. Medical journal peer review: process and bias. Pain Physician 18 (2015): E1-E14.

[22] Morey D. Richard, Chambers D. Christopher, Etchells J. Peter, Harris R. Christine, Hoekstra Rink, Lakens Daniël, Lewandowsky Stephan et al. 2016. The Peer Reviewers'

[23] Open Access Directory. 2018. Wiki. Retrieved March 6, 2018 from http://oad.simmons.edu/oadwiki/Main_Page

[24] Open Access Directory. Open Access by Nümbers. 2016. Retrieved March 6, 2018 from http://oad.simmons.edu/oadwiki/OA_by_the_numbers

[25] Openness Initiative: incentivizing open research practices through peer review. Royal Society Open Science 3, no. 1: 150547.

[26] Ross-Hellauer Tony. 2017. What is open peer review? A systematic review F1000Research 2017, 6:588 doi: 10.12688/f1000research.11369.1

[27] Stančiauskas Vilius and Banelyte, Vilte. 2017. OpenUP survey on researchers' current perceptions and practices in peer review, impact measurement and dissemination of research results. $\begin{array}{llll}\text { Retrieved } & \text { March } & 618 & \text { from }\end{array}$ https://doi.org/10.5281/zenodo.556157

[28] Tattersall Andy. 2016. Open peer review. Altmetrics: A practical guide for librarians, researchers and academics: 183

[29] The San Francisco Declaration on Research Assessment (DORA). 2012. Retrieved March 5, 2018 from http://www.ascb.org/dora/

[30] Vignoli Michela. 2017. Interim Use Case Evaluation Report OpenUP Deliverable D6.2

[31] Wilkinson D. Mark, et al. 2016. The FAIR Guiding Principles for scientific data management and stewardship. Scientific data3 http://doi.org/10.1038/sdata.2016.18

[32] Zendel Oliver, Schörghuber Matthias, and Vignoli Michela. 2017. Open Peer Review CMS Support. In Proceedings of the 13th International Symposium on Open Collaboration, p. 23. ACM, 2017 . 